\title{
Anillin is a prognostic factor and is correlated with genovariation in pancreatic cancer based on databases analysis
}

\author{
YUANHUA NIE, ZHIQIANG ZHAO, MINXUE CHEN, FULIN MA, YONG FAN, \\ YINGXIN KANG, BOXIONG KANG and CHEN WANG \\ Department of General Surgery, Lanzhou University Second Hospital, Lanzhou, Gansu 730030, P.R. China
}

Received March 12,2020; Accepted October 8, 2020

DOI: $10.3892 / 01.2020 .12368$

\begin{abstract}
Pancreatic cancer has a low survival rate globally. Anillin (ANLN) is involved in the pathogenesis of pancreatic cancer (PC). The present study used databases and reverse transcription-quantitative PCR to investigate the association between ANLN expression, clinical variables and the survival rate of patients with pancreatic cancer. Gene expression of ANLN in normal and cancer tissues was analyzed using data from The Cancer Genome Atlas, Oncomine and Gene Expression database of Normal and Tumor tissues 2 and ANOVA, and the association between ANLN mRNA expression and ANLN genovariation was analyzed using cBioPortal. The association between ANLN expression and the survival, clinical, pathological and prognostic characteristics of PC was analyzed using Kaplan-Meier (K-M) survival analysis, Kruskal Wallis and Mann Whitney-U tests, and logistic and Cox regression models. Gene Set Enrichment Analysis (GSEA) revealed the molecular pathways underpinning ANLN function in PC. Overexpression of ANLN was observed in $\mathrm{PC}$ cells (normal vs. tumor, $\mathrm{P}<0.01$ ) and tissues (normal vs. tumor, $\mathrm{P}=0.008$ ). Enhanced ANLN expression was associated with high tumor grade (grade 1 vs. grade 3, odds ratio: 5.662, $\mathrm{P}<0.001)$. However, ANLN expression was not associated with other clinical features (all $\mathrm{P}>0.05$ ). K-M analysis suggested that increased ANLN expression was associated with poor survival $(\mathrm{P}=0.002)$. Univariate and multivariate analysis revealed the ANLN is an independent prognostic factor for PC $(\mathrm{P}<0.001)$. GSEA demonstrated the p53, cell cycle, DNA replication, mismatch repair, nucleotide excision repair and
\end{abstract}

Correspondence to: Dr Chen Wang, Department of General Surgery, Lanzhou University Second Hospital, 82 Cuiyingmen, Chengguan, Lanzhou, Gansu 730030, P.R. China

E-mail: chenwang@lzu.edu.cn

Abbreviations: PC, pancreatic cancer; ANLN, anillin; GSEA, Gene Set Enrichment Analysis

Key words: ANLN, cell cycle, pancreatic cancer, The Cancer Genome Atlas, Oncomine, GSEA, cBioPortal, genovariation, prognostic factor
PC pathways were associated with low expression of ANLN. Overall, ANLN is more highly expressed in PC compared with in normal tissue, and is associated with poor differentiation. The expression of ANLN may be a novel prognostic marker of poor survival. Finally, ANLN exert its functions in PC through the p53, cell cycle, DNA replication, mismatch repair and nucleotide excision repair and pathways.

\section{Introduction}

Pancreatic cancer (PC) is a major public health problem and is the eleventh most common cancer in the world, with 458,918 new cases and 432,242 deaths in 2018 (1). Risk factors for PC include the gut microbiota, chronic pancreatitis and obesity (2). The 5-year survival rate is correlated with tumor Tumor-Node-Metastasis stage. At stage 3, the 5-year survival rate can reach $32 \%$; however, $~ 52 \%$ of patients at stage 4 have a 5 -year survival rate of only $3 \%$ (3). Hence, identifying PC in the early stages is important for an improved prognosis. Unfortunately, the symptoms of surgically resectable PC are non-specific, such as epigastric or back pain, nausea and satiety (4). As the aforementioned symptoms can be indications of benign and alterative diseases, for example acute and chronic pancreatitis and cholelithiasis, PC may be misdiagnosed and is therefore difficult to diagnose in early stages. Most patients are diagnosed in advanced stages, which makes surgery challenging, and current chemotherapy does not yield satisfactory outcomes (5-9), due to epithelial-to-mesenchymal transition, increased cancer stem cells population, (hypovascular tumor microenvironment and chemotherapy-resistance protein (10). Therefore, understanding oncogenesis, identifying a guaranteed early diagnostic marker and investigating new treatments are important to improve the diagnosis and treatment of PC.

Anillin (ANLN) was first identified by Field and Albert in 1995 (11). ANLN encodes an actin-binding protein and is an important cellular component for cytokinesis. The localization of ANLN changes during the cell cycle (12). During interphase, ANLN located at nucleus, while during mitosis, ANLN located at cell cortex (11). Furthermore, by binding to other key proteins for mitosis, such as F-actin, myosin II and septins, ANLN forms cleavage furrows (12-16). Recently, the important roles of ANLN in the proliferation and invasion of various cancer cells and tumor development have been described. Downregulating the expression of ANLN arrests cancer 
cells at the $\mathrm{G}_{2} / \mathrm{M}$ stage of mitosis, decreasing the capacity for proliferation and invasion in small-cell lung cancer. The expression of ANLN is associated with 5-year cancer-specific survival (17). In addition, correlations between ANLN and other tumors, including breast, pancreatic, prostate, bladder and colorectal cancer, have been reported (18-22). However, the expression of ANLN and its association with the clinical features of PC are unclear. Therefore, the expression of ANLN and its clinical characteristics in PC were examined in the present study. The Cancer Genome Atlas (TCGA), Oncomine, Gene Expression Ontology (GEO) and Gene Expression Database of Normal and Tumor tissues (GENT) databases were used to obtain samples and the clinical datasets were analyzed. Afterwards, Gene Set Enrichment Analysis (GSEA) was employed to analyze the pathways of ANLN and cancer development.

\section{Materials and methods}

Gene expression data and survival data resources. The Oncomine database was used to identify the expression levels of ANLN between normal tissue and cancer tissue in diverse cancer types (http://www.oncomine.org/).

Gene expression of ANLN in normal and cancerous pancreatic tissues was downloaded from TCGA database (https://portal.gdc.cancer.gov/) and the Gene Expression database of Normal and Tumor tissues 2 (GENT2) (http://gent2. appex.kr/) (23). Bioinformatics and survival data were downloaded from TCGA database. In total, 182 samples, including four normal and 178 tumor samples, with clinical information were download from TCGA database in February 2020. Gene expression of ANLN in normal $(n=106)$ and pancreatic cancer $(n=324)$ of GENT2 database was obtained from the GEO public repository using the U133Plus2 (GPL570) platform (23).

Cell lines and culture. Human pancreatic cancer cell lines, PANC-1 and AsPC-1, were purchased from CHI Scientific, Inc. Human pancreatic cancer cell line, MIA PaCa-2, and normal pancreatic cell line, hTRET-HPNE, were purchased from Shanghai Zhongqiaoxinzhou Biotech Company (https://www.zqxzbio.com/). The human pancreatic cancer cell line, CFPAC-1, was purchased from The Cell Bank of Type Culture Collection of the Chinese Academy of Sciences. hTRET-HPNE, MIA PaCa-2 and PANC-1 cells were cultured in DMEM (HyClone; Cyvita), supplemented with 10\% FBS (Gibco; Thermo Fisher Scientific, Inc.) and penicillin $\mathrm{G}(100 \mathrm{U} / \mathrm{ml})$ (Beyotime Institute of Biotechnology). AsPC-1 and CFPAC-1 cells were cultured in RPMI-1640 and IMDM (Gibco; Thermo Fisher Scientific, Inc.), respectively, with $10 \%$ FBS and penicillin $\mathrm{G}(100 \mathrm{U} / \mathrm{ml})$. All cells were maintained at $37^{\circ} \mathrm{C}$ with $5 \% \mathrm{CO}_{2}$. Cells were harvested at $80 \%$ confluence.

Reverse transcription-quantitative (RT-q)PCR. All cells were collected, and total RNA was extracted using RNAiso PLUS (Takara Bio, Inc.). According to the instructions, reverse-transcription was performed using a PrimeScript ${ }^{\mathrm{TM}} \mathrm{RT}$ reagent kit with gDNA Eraser (Takara Bio, Inc.). PCR was performed using Rotor-Gene Q (Qiagen $\mathrm{GmbH}$ ) with
TB Green ${ }^{\mathrm{TM}}$ Premix Ex Taq ${ }^{\mathrm{TM}}$ II (Tli RNase H Plus) (Takara Bio, Inc.). The PCR cycling conditions were initial denaturation at $95^{\circ} \mathrm{C}$ for $30 \mathrm{sec}$, followed by 40 of cycles of denaturation at $95^{\circ} \mathrm{C}$ for $5 \mathrm{sec}$, annealing at $55^{\circ} \mathrm{C}$ for $30 \mathrm{sec}$ and elongation at $72^{\circ} \mathrm{C}, 30 \mathrm{sec}$ and one cycle of final extension at $72^{\circ} \mathrm{C}$ for $5 \mathrm{~min}$. GAPDH was used as a housekeeping gene. ANLN gene expression was analyzed using the $2^{-\Delta \Delta C q}$ formula (24). The primer sequences are as follows: ANLN, Forward: 5'-CAAGATGTATCCAATGACT-3' and reverse: 5'-TGACTGAAGAATGAATGTT-3'; GAPDH, forward: 5'-CTCTCCACGGATCAGCTGTC-3' and reverse: 5'-CAGGGAGGACACGAAGGAT-3'. All experiments were repeated three times.

Genovariation of ANLN. cBioPortal (https://www.cbioportal. org/) was used to examine the types and frequency of ANLN alterations and methylation of ANLN in PC. The sequencing data for PC from cBioPortal was from GDAC firehose in TCGA database.

GSEA. GSEA (https://www.gsea-msigdb.org/gsea/datasets. jsp) was performed to conduct Kyoto Encyclopedia of Genes and Genomes (KEGG) pathway biological process enrichment analysis to explore the gene sets.

Statistical analysis. $\mathrm{P}<0.0001$, fold-change $>2$ and top $10 \%$ gene rank were used as the significance threshold values for the Oncomine database. Data from TCGA and GEO databases were analyzed using R (version 3.6.2) (25) and GENT2, respectively. Spearman's correlation analysis was utilized to determine the relationship between the levels of ANLN mRNA expression and genovariation of ANLN. Gene expression levels over the median were considered high expression and those below the median were low expression (median value, 3.648). To examine the association between 5-year survival rate and ANLN expression, K-M survival analysis and log rank test was used. Comparison of biological information and ANLN expression between two groups was performed using Mann Whitney U tests, while comparison between three groups or more was performed using Kruskal Wallis and Dunn's post hoc tests. Logistic regression was used to analyze the association between clinical variables and ANLN expression. Univariate and multivariate Cox regression was used to determine whether ANLN expression level was a prognostic factor. Other differences between two groups was analyzed using unpaired or paired Student's t-tests as appropriate, whereas differences between three or more groups was performed by ANOVA followed by Dunnett's post hoc test. $\mathrm{P}<0.05$ was considered to indicate a statistical significant difference.

\section{Results}

Gene expression of ANLN is increased in PC. The Oncomine database revealed that the expression of ANLN was increased in bladder, breast, cervical, colorectal, esophageal, gastric, head and neck, liver, lung, lymphoma, ovarian and pancreatic cancer (Fig. 1A). TCGA database was also searched and obtained similar results for PC (Fig. 1B). ANLN expression in normal pancreas tissue was much lower than in pancreatic tumor tissue $(\mathrm{P}=0.008)$. To rule out bias, each adjacent normal 
A

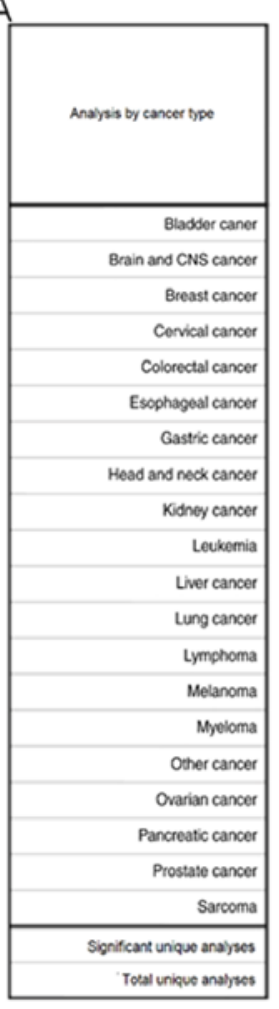

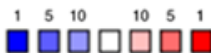

B

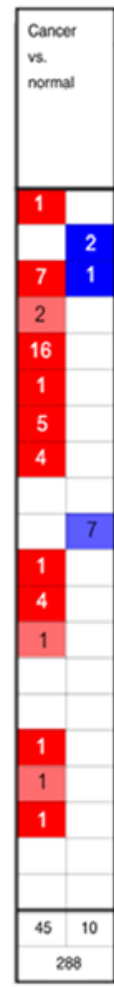

$B$
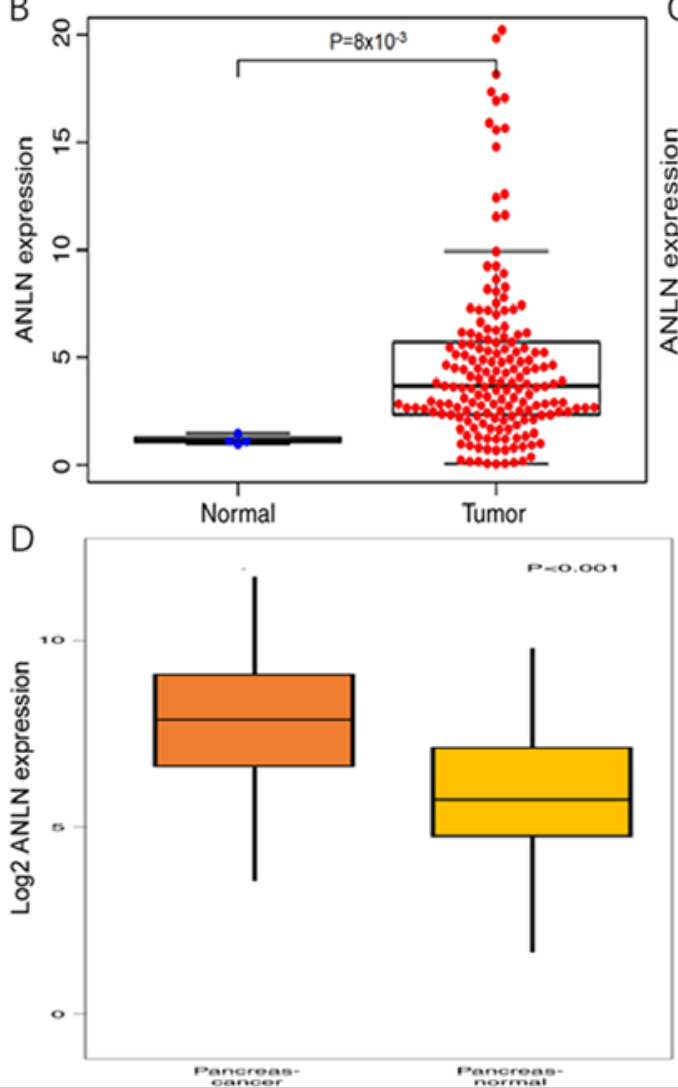

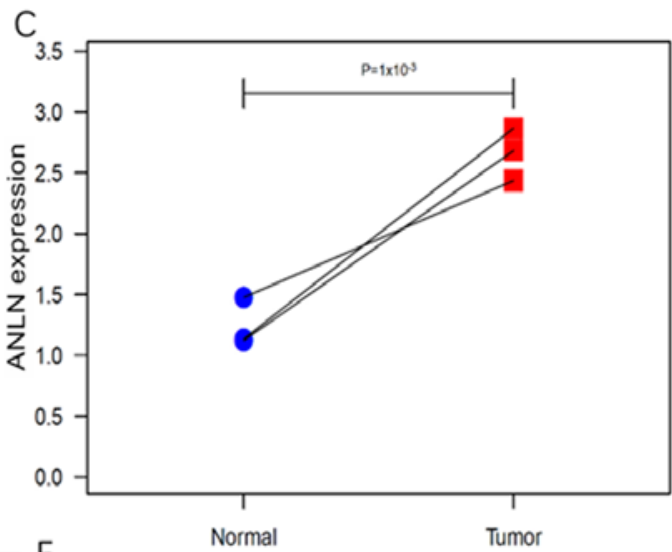

E

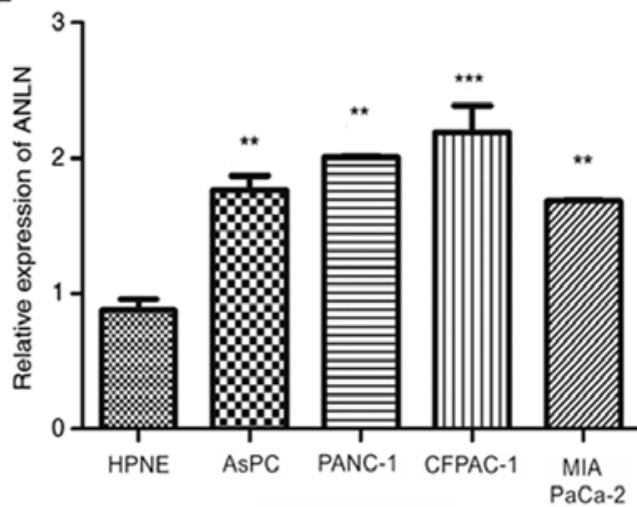

Figure 1. ANLN expression in pancreatic cancer and normal tissue. (A) Expression of ANLN in different cancer types in Oncomine database. (B) Expression of ANLN in normal and tumor tissues in TCGA database. (C) Expression of ANLN in paired normal and tumor tissues from TCGA database. (D) Expression of ANLN in normal and tumor tissues in GENT2. (E) Expression of ANLN mRNA in pancreatic cancer and normal pancreatic cells detected using reverse transcription-quantitative PCR. ${ }^{* *} \mathrm{P}<0.01,{ }^{* * *} \mathrm{P}<0.001$ vs. HPNE. ANLN, anillin; TCGA, The Cancer Genome Atlas.

Table I. Summary of genovariation of ANLN in pancreatic cancer.

\section{Alterations}

Frequency, n (\%)

$\begin{array}{lr}\text { Mutation } & 1(0.54) \\ \text { Amplification } & 1(0.54) \\ \text { mRNA high } & 15(8.11) \\ \text { Multiple alteration } & 1(0.54) \\ \text { Total } & 18(9.73)\end{array}$

sample was paired with its tumor tissue and the expression of ANLN was analyzed. As expected, ANLN was more highly expressed in tumor tissues compared with in normal tissues $(\mathrm{P}=0.001$; Fig. $1 \mathrm{C})$. As the quantity of normal tissue in TCGA database is notably different from tumor tissue and there was no more data for normal and tumor tissues in TCGA, the GEO database was used to verify these results. The $\log 2$ fold-change was 1.849 , ANLN was significantly more highly expressed in tumor tissue compared with in normal tissue ( $\mathrm{P}<0.001$; Fig. 1D). Moreover, the differences in ANLN mRNA expression between normal pancreas cell line and each of PC cell lines were analyzed. RT-PCR indicated that all cancer cell lines presented higher expression levels of ANLN compared with the normal cell line $(\mathrm{P}<0.01$; Fig. 1E).
Mutations in ANLN are not common, and mRNA expression of $A N L N$ is related to methylation. In total, there were 186 samples from 185 patients in cBioPortal. As shown in Table I, only $9.73 \%$ of patients exhibited genetic alterations in ANLN. Mutation, amplification and multiple alterations occurred in one case each. mRNA levels were high in 15 cases, with a frequency of $8.11 \%$.

The patient with multiple alterations in the ANLN gene had three missenses mutations dispersed at different locations that caused changes in the protein (Fig. 2A). Most patients had no mutations. As illustrated in Fig. 2B, it was concluded that there was no significant difference in the mRNA expression of ANLN among the different genotypes. Additionally, the methylation and mRNA expression of ANLN was analyzed. Spearman's correlation analysis showed a weak but statistically significant negative correlation between gene methylation and mRNA expression of ANLN, with the coefficient and P-value equaling -0.22 and 0.002664 , respectively (Fig. 2C).

High expression of ANLN predicts poor survival and grade, representing an independent survival index. As shown in Fig. 3A, high expression levels of ANLN predicted poor survival $(\mathrm{P}=0.002)$. ANLN expression was not associated with some clinical features, such as stage ( $\mathrm{P}=0.229$; Fig. 3B), lymph node metastasis $(\mathrm{P}=0.524$; Fig. $3 \mathrm{E})$, distant metastasis $(\mathrm{P}=0.726$; Fig. 3F) and tumor size $(\mathrm{P}=0.127$; Fig. 3D) but was associated with higher grade $(\mathrm{P}<0.01$; Fig. $3 \mathrm{C})$. Logistic 

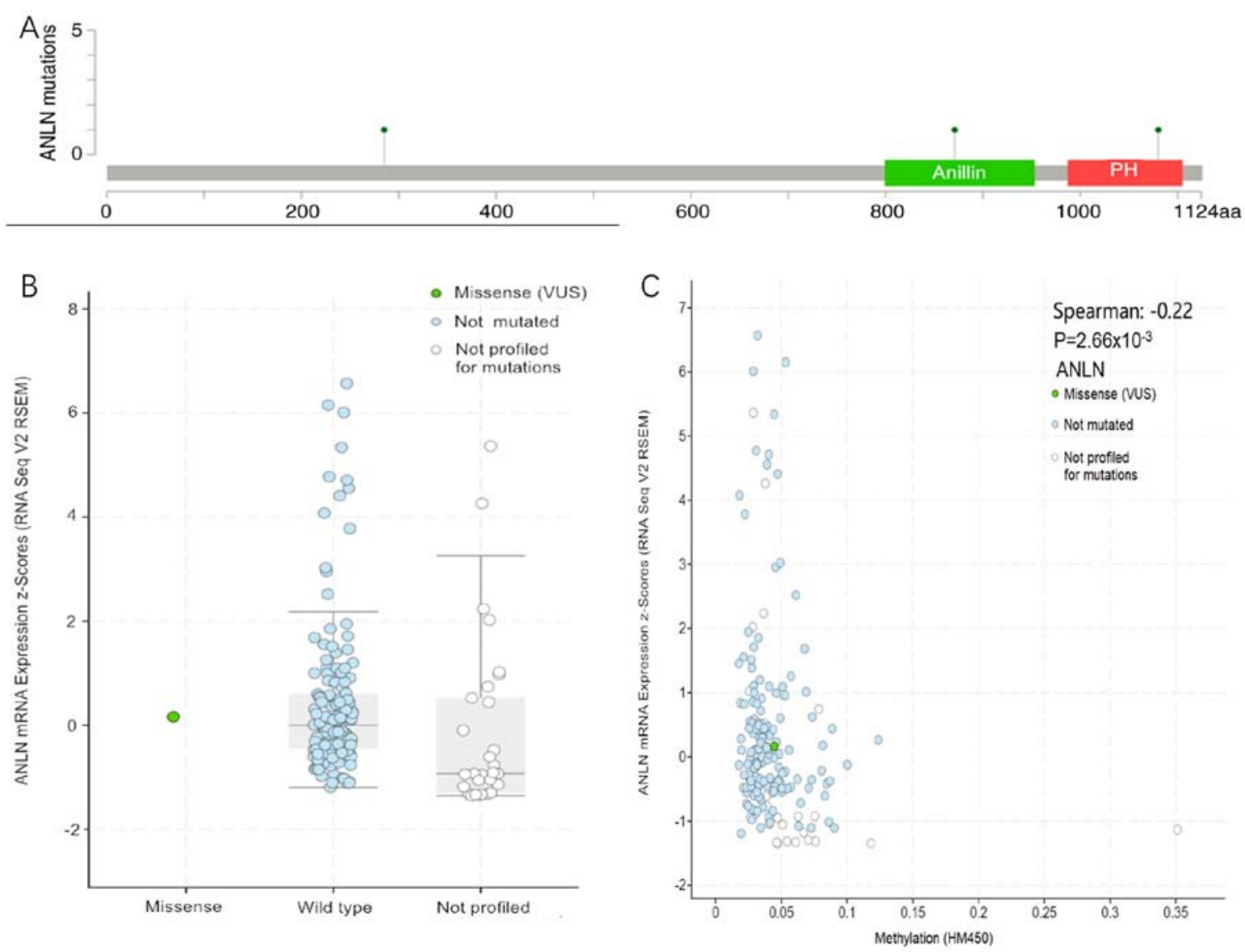

Figure 2. Genovariation of ANLN in pancreatic cancer. (A) Location of each mutation. (B) Levels of ANLN mRNA expression in mutant and normal ANLN were not significantly different. (C) Significant weak negative correlation between ANLN mRNA expression and gene methylation. ANLN, anillin; PH, Pleckstrin Homolgy; VUS, Variants of Uncertain Significance; seq, sequencing; RSEM, RNA-Seq by Expectation Maximization.


Figure 3. Association between ANLN expression and clinical features. (A) Correlation between ANLN expression with 5-year survival rate. Association between ANLN expression with (B) stage, (C) tumor grade, (D) tumor size, (E) lymph node metastasis and (F) distant metastasis. ANLN, anillin; T, tumor; $\mathrm{G}$, grade; $\mathrm{N}$, node; $\mathrm{M}$, metastasis. 
Table II. Association between the clinical characteristics and anillin expression using logistic regression.

\begin{tabular}{lccc}
\hline Clinical characteristic & Total & Odds ratio (95\% CI) & P-value \\
\hline Grade, 3 vs. 1 & 182 & $5.662(3.590-35.527)$ & $4.85 \times 10^{-5}$ \\
Stage, 4 vs. 1 & 182 & $1.370(0.430-88.643)$ & 0.262 \\
Lymph node metastasis, positive vs. negative & 180 & $1,187(0.612-2.315)$ & 0.612 \\
Distant metastasis, positive vs. negative & 90 & $3.237(0.395-66.980)$ & 0.218 \\
\hline
\end{tabular}

CI, confidence interval.

Table III. Relationship between clinical characteristics and overall survival in pancreatic cancer using univariate and multivariate Cox regression.

\begin{tabular}{|c|c|c|c|c|}
\hline \multirow[b]{2}{*}{ Clinical characteristic } & \multicolumn{2}{|c|}{ Univariate analysis } & \multicolumn{2}{|c|}{ Multivariate analysis } \\
\hline & HR $(95 \% \mathrm{CI})$ & P-value & HR $(95 \% \mathrm{CI})$ & P-value \\
\hline Age & $1.028(1.005-1.051)$ & 0.016 & $1.024(1.001-1.047)$ & 0.041 \\
\hline Sex, male vs. female & $0.781(0.509-1.200)$ & 0.259 & $0.891(0.570-1.395)$ & 0.615 \\
\hline Grade & $1.331(0.981-1.806)$ & 0.066 & $1.160(0.855-1.574)$ & 0.341 \\
\hline Stage & $1.294(0.862-1.942)$ & 0.214 & $0.994(0.521-1.895)$ & 0.984 \\
\hline Tumor size & $1.624(0.977-2.701)$ & 0.061 & $1.266(0.638-2.513)$ & 0.500 \\
\hline Lymph node metastasis, positive vs. negative & $2.258(1.308-3.898)$ & 0.003 & $1.820(1.028-3.220)$ & 0.040 \\
\hline ANLN & $1.107(1.061-1.156)$ & 0.000 & $1.090(1.054-1.139)$ & $<0.001$ \\
\hline
\end{tabular}

ANLN, anillin; HR, hazard ratio; CI, confidence interval.

Table IV. Gene sets enriched in different ANLN phenotypes.

\begin{tabular}{llrrr}
\hline ANLN expression phenotype & \multicolumn{1}{c}{ Gene set name } & NES & Nom P-value & FDR q-value \\
\hline High expression & neuroactive_ligand_receptor_interaction & 1.826 & $<0.001$ & 0.123 \\
Low expression & cell_cycle & -2.255 & $<0.001$ & $<0.001$ \\
& P53_signaling_pathway & -2.158 & $<0.001$ & $<0.001$ \\
& DNA_replication & -1.879 & $<0.001$ & 0.016 \\
& Mismatch_repair & -1.927 & $<0.001$ & 0.011 \\
& Nucleotide_excision_repair & -1.872 & $<0.001$ & 0.016 \\
& Pancreatic_cancer & -1.743 & 0.008 & 0.031
\end{tabular}

ANLN, anillin; NES, Normalized Enrichment Score; FDR, false discovery rate; Nom, Nominal.

regression analysis revealed the same conclusion; high ANLN expression is significantly associated with worse grade [grade 3 vs. 1 odds ratio (OR): 5.662; 95\% confidence interval $(\mathrm{CI}), 3.590,35.527 ; \mathrm{P}<0.001]$ while the other clinical information including stage, lymph node metastasis and distant metastasis was not related to ANLN expression with $\mathrm{P}=0.262$, 0.612 and 0.218 , respectively (Table II).

Univariate analysis demonstrated that higher expression of ANLN indicated worse prognosis [hazard ratio (HR): 1.107, 95\% CI (1.061, 1.156); P<0.001; Table III). Multivariate analysis also confirmed that ANLN was an independent factor for prognosis (HR: 1.090, 95\% CI $(1.043,1.139), \mathrm{P}<0.001)$ The age and lymph node metastasis are another prognostic factors with $\mathrm{P}<0.05$. Univariate and multivariate analysis confirmed other factors including sex, grade, stage and tumor size were not related to prognosis (Table III).

GSEA identified ANLN-relevant pathways. GSEA was used to identify KEGG signaling pathways associated with ANLN in PC.GSEA demonstrated that high ANLN expression was correlated with the 'neuroactive ligand receptor interaction pathway' [Normalized Enrichment Score (NES): 1.826, nominal $\mathrm{P}<0.001$ Fig. S1], while low expression of ANLN was correlated with various pathways associated with cancer development, such as 'p53 signaling' (Fig. S2), the 'cell cycle' (Fig, S3), 'DNA replication' (Fig. S4), 'mismatch repair’ (Fig. S5), 'nucleotide 
excision repair' (Fig. S6) and 'PC pathways' (Fig. S7) pathway (Table IV).

\section{Discussion}

Upregulated expression of ANLN and its function have been reported in numerous cancer types, including PC $(19,20,22,26-30)$. Some studies have also demonstrated that PC progression is attributed to ANLN by enhancing EZH2 expression and manipulating the microRNA-218-5p/LIM and SH3 domain protein 1 signaling axis (31). The present bioinformatics analysis revealed that the expression of ANLN was elevated in PC, and increased expression is associated with higher grade. Moreover, ANLN represents was an independent predictor of prognosis. In addition, GSEA reported that ANLN was associated with 'p53', 'cell cycle', 'DNA replication', 'mismatch repair', 'nucleotide excision repair' and 'PC pathways'.

Previous studies have demonstrated that ANLN is overexpressed in pancreatic ductal adenocarcinoma and that its expression is correlated with differentiation in bladder cancer $(20,32)$. These are consistent with the present results, in which ANLN expression was elevated in PC tissues compared with normal pancreatic tissues. TCGA database showed that expression of ANLN was increased in PC tissue. Due to the notable difference in the number of tissues between cancer and normal specimens, paired and GEO database analyses were performed and reached the same conclusion. In addition to the database analysis, RT-qPCR also confirmed that ANLN was more highly expressed in PC cells.

Subsequently, databases were used to analyze whether elevated ANLN expression affects the biological features of PC. Enhanced expression of ANLN was correlated with advanced differentiation. However, Wang et al (31) reported that the levels of ANLN expression were correlated with tumor size, differentiation, Tumor-Node-Metastasis stage, lymph node metastasis and distant metastasis, which may be mediated by cell-cell adhesion-related genes, for example the catenin $\beta-1$ gene promoting the proliferation of hepatoblastoma (33). The results of Wang et al (31)are slightly different from the present data. The quantity of original data downloaded from TCGA database might not be sufficient, which could have contributed to this difference. Some clinical data are still unknown in TCGA database, for instance nearly half of patients' distant metastasis data was unclear.

According to the present univariate and multivariate Cox regression results, it was concluded that ANLN is an independent prognostic factor for PC. There is a possibility that ANLN may impact prognosis by contributing to cancer cell propagation, invasion, aggressiveness and chemotherapy sensitivity. Previous studies also support this hypothesis, for example, when treated with chemotherapy, patients of breast cancer with high expression of ANLN had worse prognosis (21). Wang et al (31) reported that knockdown of ANLN inhibits the invasion of pancreatic cancer cells and high expression of ANLN is related to poor prognosis of PC. In addition, another study demonstrated that high expression of ANLN increases the migration of non-small cell lung cancer cells and is associated with a worse prognosis of (17). Chemotherapy sensitivity was not confirmed in the bioinformatics analysis, because treatment for each patient was not mentioned in TCGA database. Hence, the study (21) was reviewed and how ANLN affects treatment was identified. Knockdown ANLN decreases the expression of cyclin D1, stalls the cell cycle in the $\mathrm{G}_{2} / \mathrm{M}$ phase and inhibits breast cancer cell proliferation. Therefore, the study demonstrated that high expression of ANLN limits the efficacy of chemotherapy in breast cancer; however, the reason for this is still unclear (21). However, a direct association between ANLN and chemotherapy in PC is yet to be reported.

Mutation of ANLN is uncommon, with only a few patients $(9.73 \%)$ presenting with ANLN gene alterations in the present study. Among all alterations, increased mRNA was most common and a significant correlation was identified between methylation and ANLN mRNA expression. Methylation at different positions causes diverse results, including regulating gene expression (34). However, the present study did not identify where ANLN methylation occurs or whether the high levels of ANLN mRNA are attributed to alterations in methylation. Further investigation needed to resolve the exact association between methylation and ANLN mRNA expression.

From GSEA, high expression of ANLN was correlated with the 'neuroactive ligand receptor interaction pathway'. However, to the best of our knowledge no study has investigated this relationship in PC. Low expression of ANLN was associated with 'p53 signaling', 'cell cycle', 'DNA replication', 'mismatch repair', 'nucleotide excision repair' and 'PC pathways'. Various reports have demonstrated that ANLN serves an essential role as a regulator in the cell cycle pathway during which eukaryotic cells divide $(12,35)$. Chromosome-derived Ran-GTP signals decrease local ANLN expression in the cortex of proliferating cells, which causes asymmetric membrane elongation during mitosis (36). As reported, under pathological circumstances, the development of multiple cancer types can be caused by genetic mutations and dysregulated mitotic proliferation (37). In other words, abnormal expression of ANLN during the cell cycle may promote the development of cancer. Further studies, including investigation of the relative pathways and treatment associated with ANLN expression, are needed in PC.

\section{Acknowledgements}

Not applicable.

\section{Funding}

This study was funded by The Cuiying Innovation Project of Science and Technology of Lanzhou University (grant no. CY2017-BJ04).

\section{Availability of data and materials}

The datasets used and/or analyzed during the present study are available from the corresponding author on reasonable request. Additional datasets generated and/or analyzed during the current study are available in The Cancer Genome Atlas (https://portal.gdc.cancer.gov/), Oncomine (http://www. oncomine.org/), Gene Expression database of Normal and Tumor tissues 2 (GENT2) (http://gent2.appex.kr/), cBioPortal 
(https://www.cbioportal.org/) and GSEA (https://www. gsea-msigdb.org/gsea/datasets.jsp) repositories.

\section{Authors' contributions}

CW conceived and designed the study. YN, ZZ, MC, FM, YF, $\mathrm{YK}$ and $\mathrm{BK}$ obtained the bioinformation data and analyzed the data. YN wrote the manuscript. All authors read and approved the final manuscript.

\section{Ethics approval and consent to participate}

Not applicable.

\section{Patient consent for publication}

Not applicable.

\section{Competing interests}

The authors declare that they have no competing interests.

\section{References}

1. Bray F, Ferlay J, Soerjomataram I, Siegel RL, Torre LA and Jemal A: Global cancer statistics 2018: GLOBOCAN estimates of incidence and mortality worldwide for 36 cancers in 185 countries. CA Cancer J Clin 68: 394-424, 2018.

2. McGuigan A, Kelly P, Turkington RC, Jones C, Coleman HG and McCain RS: Pancreatic cancer: A review of clinical diagnosis, epidemiology, treatment and outcomes. World J Gastroenterol 24: 4846-4861, 2018.

3. American Cancer Society: Cancer Facts \& Figures 2020. American Cancer Society, Inc., Atlanta, GA, 2020. https://www. cancer.org/research/cancer-facts-statistics/all-cancer-facts-figures/ cancer-facts-figures-2020.html. Accessed February, 2020.

4. Mizrahi JD, Surana R, Valle JW and Shroff RT: Pancreatic cancer. Lancet 395: 2008-2020, 2020.

5. Avgerinos DV and Björnsson J: Malignant neoplasms: Discordance between clinical diagnoses and autopsy findings in 3,118 cases. Apmis 109: 774-780, 2010.

6. Bosetti C, Bertuccio P, Negri E, La Vecchia CL, Zeegers MP and Boffetta P: Pancreatic cancer: Overview of descriptive epidemiology. Mol Carcinog 51: 3-13, 2012.

7. Lambe M, Eloranta S, Wigertz A and Blomqvist P: Pancreatic cancer; reporting and long-term survival in Sweden. Acta Oncol 50: 1220-1227, 2011.

8. Oberstein PE and Olive KP: Pancreatic cancer: Why is it so hard to treat? Therap Adv Gastroenterol 6: 321-337, 2013.

9. Sens MA, Zhou XD, Weiland T and Cooley AM: Unexpected neoplasia in autopsies: Potential implications for tissue and organ safety. Arch Pathol Lab Med 133: 1923-1931, 2009.

10. Uzunparmak B and Sahin IH: Pancreatic cancer microenvironment: A current dilemma. Clin Transl Med 8, 2019.

11. Field CM and Alberts BM: Anillin, a contractile ring protein that cycles from the nucleus to the cell cortex. J Cell Biol 131: $165-178,1995$.

12. Oegema K, Savoian MS, Mitchison TJ and Field CM: Functional analysis of a human homologue of the drosophila actin binding protein anillin suggests a role in cytokinesis. J Cell Biol 150 539-552, 2000

13. Gould GW: Animal cell cytokinesis: The role of dynamic changes in the plasma membrane proteome and lipidome. Semin Cell Dev Biol 53: 64-73, 2016.

14. Goyal A, Takaine M, Simanis V and Nakano K: Dividing the spoils of growth and the cell cycle: The fission yeast as a model for the study of cytokinesis. Cytoskeleton (Hoboken) 68: 69-88, 2011

15. D'Avino PP: How to scaffold the contractile ring for a safe cytokinesis-lessons from Anillin-related proteins. J Cell Sci 122 1071-1079, 2009.

16. Song K, Russo G and Krauss M: Septins as modulators of endo-lysosomal membrane traffic. Front Cell Dev Biol 4: 124, 2016.
17. Suzuki C, Daigo Y, Ishikawa N, Kato T, Hayama S, Ito T, Tsuchiya E and Nakamura Y: ANLN plays a critical role in human lung carcinogenesis through the activation of RHOA and by involvement in the phosphoinositide 3-kinase/AKT pathway. Cancer Res 65: 11314-11325, 2005.

18. Wang G, Shen W, Cui L, Chen W, Hu X and Fu J: Overexpression of Anillin (ANLN) is correlated with colorectal cancer progression and poor prognosis. Cancer Biomark 16: 459-465, 2016.

19. Zeng S, Yu X, Ma C, Song R, Zhang Z, Zi X, Chen X, Wang Y, $\mathrm{Yu} \mathrm{Y}$, Zhao J, et al: Transcriptome sequencing identifies ANLN as a promising prognostic biomarker in bladder urothelial carcinoma. Sci Rep 7: 3151, 2017.

20. Olakowski M, Tyszkiewicz T, Jarzab M, Król R, Oczko-WojciechowskaM,KowalskaM,KowalM,GalaGM,KajorM, Lange D, et al: NBL1 and anillin (ANLN) genes over-expression in pancreatic carcinoma. Folia Histochem Cytobiol 47: 249-255, 2009.

21. Wang Z, Chen J, Zhong MZ, Huang J, Hu YP, Feng DY, Zhou ZJ, Luo X, Liu ZQ, Jiang WZ and Zhou WB: Overexpression of ANLN contributed to poor prognosis of anthracycline-based chemotherapy in breast cancer patients. Cancer Chemother Pharmacol 79: 535-543, 2017.

22. Tamura K, Furihata M, Tsunoda T, Ashida S, Takata R, Obara W, Yoshioka H, Daigo Y, Nasu Y, Kumon H, et al: Molecular features of hormone-refractory prostate cancer cells by genome-wide gene expression profiles. Cancer Res 67: 5117-5125, 2007.

23. Park SJ, Yoon BH, Kim SK and Kim SY: GENT2: An updated gene expression database for normal and tumor tissues. BMC Med Genomics 12 (Suppl 5): 101, 2019.

24. Livak KJ and Schmittgen TD: Analysis of relative gene expression data using real-time quantitative PCR and the 2(-Delta Delta C(T)) method. Methods 25: 402-408, 2001.

25. Team R: RStudio: Integrated Development for R, 2015. RStudio, Inc., Boston, MA, 2015. http://www.rstudio.com.

26. Long X, Zhou W, Wang Y and Liu S: Prognostic significance of ANLN in lung adenocarcinoma. Oncol Lett 16: 1835-1840, 2018.

27. Sadi AM, Wang DY, Youngson BJ, Miller N, Boerner S, Done SJ and Leong WL: Clinical relevance of DNA microarray analyses using archival formalin-fixed paraffin-embedded breast cancer specimens. Bmc Cancer 11: 253, 2011.

28. Wang S, Mo Y, Midorikawa K, Zhang Z, Huang G, Ma N, Zhao W, Hiraku Y, Oikawa S and Murata M: The potent tumor suppressor miR-497 inhibits cancer phenotypes in nasopharyngeal carcinoma by targeting ANLN and HSPA4L. Oncotarget 6: 35893-35907, 2015.

29. Weinberger P, Ponny SR, Xu H, Bai S, Smallridge R, Copland J and Sharma A: Cell Cycle M-Phase genes are highly upregulated in anaplastic thyroid carcinoma. Thyroid 27: 236-252, 2016.

30. Xia L, Su X, Shen J, Meng Q, Yan J, Zhang C, Chen Y, Wang H and $\mathrm{Xu} \mathrm{M}$ : ANLN functions as a key candidate gene in cervical cancer as determined by integrated bioinformatic analysis. Cancer Manag Res 10: 663-670, 2018.

31. Wang A, Dai H, Gong Y, Zhang C, Shu J, Luo Y, Jiang Y, Liu W and Bie P: ANLN-induced EZH2 upregulation promotes pancreatic cancer progression by mediating miR-218-5p/LASP1 signaling axis. J Exp Clin Cancer Res 38: 347, 2019.

32. Zeng S: The mechanism of ANLN in the carcinogenesis of bladder cancer. PhD dissertation, Chinese People's Liberation Army Naval Medical University Shanghai 211 Engineering College, Shanghai, China, 2017.

33. Liu L, Wang J, Sun G, Wu Q, Ma J, Zhang X, Huang N, Bian Z, Gu S, $\mathrm{Xu}$ M, et al: $\mathrm{m}^{6} \mathrm{~A} \mathrm{mRNA}$ methylation regulates CTNNB1 to promote the proliferation of hepatoblastoma. Mol Cancer 18: 188, 2019.

34. Ghoshal K, Majumder S, Li Z, Dong X and Jacob ST: Suppression of metallothionein gene expression in a rat hepatoma because of promoter-specific DNA methylation. J Biol Chem 275: 539-547, 2000.

35. Nigg EA: Mitotic kinases as regulators of cell division and its checkpoints 2: 21-32, 2001

36. Kiyomitsu $\mathrm{T}$ and Cheeseman IM: Cortical dynein and asymmetric membrane elongation coordinately position the spindle in anaphase. Cell 154: 391-402, 2013.

37. Brinkley BR: Managing the centrosome numbers game: From chaos to stability in cancer cell division. Trends Cell Biol 11: 18-21, 2001.

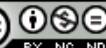

This work is licensed under a Creative Commons Attribution-NonCommercial-NoDerivatives 4.0 International (CC BY-NC-ND 4.0) License. 